\title{
The Asymptotic Stability of a Nonstationary System with Delay
}

\author{
B. G. Grebenshchikov ${ }^{1 *}$ \\ ${ }^{1}$ Ural Federal University, ul. Mira 19, Ekaterinburg, 620002 Russia ${ }^{1}$ \\ Received May 2, 2012
}

\begin{abstract}
In this paper we study the exponential stability of a linear system of differential equations with a constant delay such that the right-hand side of one of its subsystems contains an exponential multiplier. We establish sufficient conditions for the existence of an asymptotically periodic solution to the inhomogeneous system.
\end{abstract}

DOI: $10.3103 / \mathrm{S} 1066369 \times 13080033$

Keywords and phrases: asymptotic stability, exponential bound, asymptotically periodic solution.

\section{STABILITY OF NONSTATIONARY HOMOGENEOUS SYSTEMS}

Consider the following linear homogeneous system with a constant delay:

$$
\begin{gathered}
d x(t) / d t=A_{1}(t) x(t)+A_{2}(t) x(t-\sigma)+B_{1}(t) y(t)+B_{2}(t) y(t-\sigma), \\
d y(t) / d t=\theta_{0} e^{t}\left[A_{3} x(t)+A_{4} x(t-\sigma)+B_{3} y(t)+B_{4} y(t-\sigma)\right], \\
t \geq 0, \quad \theta_{0}>0, \sigma=\mathrm{const}, \sigma>0 .
\end{gathered}
$$

Here $A_{i}(t)$ and $B_{i}(t)(i=1,2)$ are periodic (with a period $\left.0<T \leq \sigma\right)$ continuously differentiable $m \times m$-matrices, $A_{k}$ and $B_{k}(k=3,4)$ are constant $m \times m$-matrices, while $x(t)$ and $y(t)$ are $m$ dimensional vector functions with respect to (the argument) $t$.

We define the norm of a vector $w=\left\{w_{j}\right\}^{(\top)}$ (here $w_{j}$ are components of the vector $w$, and $\left(^{\top}\right.$ ) is the transposition sign) by the equality $\|w\|=\sum_{j=1}^{m}\left|w_{j}\right|$; we do the norm of a matrix $D=\left\{d_{i j}\right\}$ $(i, j=1, \ldots, m)$ in accordance with the norm of a vector ([1], P. 12), namely, $\|D\|=\max _{j} \sum_{i}\left|d_{i j}\right|$.

Earlier we studied properties of a system with constant matrices $A_{i}^{0}$ and $B_{i}^{0}(i=1,2)$ in papers [2-4]. Note that one can obtain system (1.1) by replacing the time variable $t=\ln \left(\frac{\theta}{\theta_{0}}\right)$ in the following system with a linear delay:

$$
\begin{gathered}
d \widehat{x}(\theta) / d \theta=\frac{1}{\theta}\left[\widehat{A}_{1}(\theta) \widehat{x}(\theta)+\widehat{A}_{2}(\theta) \widehat{x}(\mu \theta)+\widehat{B}_{1}(\theta) \widehat{y}(t)+\widehat{B}_{2}(\theta) \widehat{y}(\mu \theta)\right], \\
d \widehat{y}(\theta) / d \theta=A_{3} \widehat{x}(\theta)+A_{4} \widehat{x}(\mu \theta)+B_{3} \widehat{y}(\theta)+B_{4} y(\mu \theta), \\
\theta \geq \theta_{0}>0, \quad \sigma=-\ln (\mu), \quad 0<\mu<1 .
\end{gathered}
$$

On an initial set $\left[\mu \theta_{0}, \theta_{0}\right]$ we define an initial vector function $\widehat{\phi}(\theta)$. We can solve this system by successive integration on segments $h_{n}(\theta)=\left(\frac{\theta_{0}}{\mu^{n}}, \frac{\theta_{0}}{\mu^{n+1}}\right]$. This method does not allow one to establish asymptotic properties of the system under consideration.

\footnotetext{
${ }^{1}$ E-mail: ablozhnikov@yandex.ru

*E-mail: ablozhnikov@yandex.ru.
} 
As was proved earlier, solution $\left\{x^{0}(t), y^{0}(t)\right\}^{\top}$ to the system

$$
\begin{gathered}
d x^{0}(t) / d t=A_{1}^{0} x^{0}(t)+A_{2}^{0} x^{0}(t-\sigma)+B_{1}^{0} y^{0}(t)+B_{2}^{0} y^{0}(t-\sigma), \\
d y^{0}(t) / d t=\theta_{0} e^{t}\left[A_{3} x^{0}(t)+A_{4} x^{0}(t-\tau)+B_{3} y^{0}(t)+B_{4} y^{0}(t-\tau)\right], \quad t \geq 0,
\end{gathered}
$$

is exponentially stable, and the system itself is stable in the first approximation, if all the following conditions are fulfilled:

1) roots $\lambda$ of the characteristic equation

$$
\left|A_{1}^{0}+A_{2}^{0} e^{-\lambda \sigma}-\lambda E\right|=0
$$

have negative real parts, i.e.,

$$
\operatorname{Re} \lambda<-\beta_{1}, \beta_{1}=\text { const, } \beta_{1}>0 ;
$$

2) eigenvalues $\nu$ of the matrix $B_{3}$ have negative real parts, i.e.,

$$
\operatorname{Re} \nu<-\beta_{2}, \beta_{2}=\text { const, } \beta_{2}>0 ;
$$

3) roots $p$ of the characteristic equation

$$
\left|B_{3}+B_{4} e^{-p \sigma}-\left(A_{3}+A_{4} e^{-p \sigma}\right)\left(A_{1}^{0}+A_{2}^{0} e^{-p \sigma}-p E\right)^{-1}\left(B_{1}^{0}+B_{2}^{0} e^{-p \sigma}\right)\right|=0
$$

satisfy the inequality

$$
\operatorname{Re} \nu<-\beta_{3}, \beta_{3}=\text { const, } \beta_{3}>0 .
$$

In the paper [2] with the help of the Laplace transform [5] we prove that under conditions (1.3)(1.5) solution $x^{0}\left(t, \phi_{1}(\eta)\right), y^{0}\left(t, \phi_{2}(\eta)\right)$ to system (1.2) (defined by the initial vector function $\phi^{(\top)}(\eta)=$ $\left.\left\{\phi_{1}(\eta), \phi_{2}(\eta)\right\}: x(\eta)=\phi_{1}(\eta), y(\eta)=\phi_{2}(\eta), \eta \in[-\sigma, 0]\right)$ satisfies the bound

$$
\begin{gathered}
\left\|x^{0}(t)\right\|+\left\|y^{0}(t)\right\| \leq M_{0} e^{-\beta_{0} t}\left[\sup _{\eta}\left\|\phi_{1}(\eta)\right\|+\sup _{\eta}\left\|\phi_{2}(\eta)\right\|\right], \quad t \geq 0, \\
M_{0}=\text { const, } M_{0}>1, \quad \beta_{0}=\min \left\{\beta_{1}, \beta_{3}\right\}-\bar{\varepsilon}
\end{gathered}
$$

$\left(\beta_{0}>0\right.$ with sufficiently small positive $\left.\bar{\varepsilon}\right)$.

Let us define a solution to the initial system (1.1) by the same vector function $\phi(\eta)$ (we set $\sup \left\|\phi_{1}(\eta)\right\|+\sup \left\|\phi_{2}(\eta)\right\|<\delta_{0}$, while $\delta_{0}$ is a sufficiently small positive number). In order to better understand properties of the initial system, we proceed from system (1.1) (a finite system of differential equations with a delay on an infinite time interval) to a countable system of ordinary differential equations given on a finite time interval $[0, \sigma]$. To this end, we set $x_{n+1}(t)=x(n \sigma+t)$ and $y_{n+1}(t)=y(n \sigma+t)$ $(n=1,2 \ldots), t \in[0, \sigma])$. We get the following totality of two subsystems:

$$
\begin{gathered}
d x_{n+1}(t) / d t=A_{1}(t) x_{n+1}(t)+A_{2}(t) x_{n}(t)+B_{1}(t) y_{n+1}(t)+B_{2}(t) y_{n}(t), \\
\varepsilon_{n} d y_{n+1}(t) / d t=e^{t}\left[A_{3} x_{n+1}(t)+A_{4} x_{n}(t)+B_{3} y_{n+1}(t)+B_{4} y_{n}(t)\right], \\
t_{0} \leq t \leq t_{0}+\sigma, \quad \varepsilon_{n}=e^{-n \sigma} / \theta_{0}=\mu^{n} / \theta_{0}, \quad x_{0}(t)=\phi_{1}(t-\sigma), \quad y_{0}=\phi_{2}(t-\sigma),
\end{gathered}
$$

satisfying boundary conditions ([5], P. 103)

$$
x_{n+1}(0)=x_{n}(\sigma), y_{n+1}(0)=y_{n}(\sigma) .
$$

Thus, we have reduced the solution of system (1.1) to the successive integration of a "differentialdifference" system in the space of continuous vector functions given on the segment $[0, \sigma]$. Let us mention some properties of subsystem (1.8). Since $\varepsilon_{n} \rightarrow 0$ as $n \rightarrow \infty$, subsystem (1.8) is singularly perturbed [2,3], consequently, the solution set of system $(1.7),(1.8)$ contains slow variables $\left(x_{n}(t)\right)$ and fast ones $\left(y_{n}(t)\right)$. Moreover, with sufficiently large $n$, just the presence of a small parameter $\varepsilon_{n}$ allows us to consider the asymptotic behavior of subsystem (1.7) with periodic matrices $A_{i}(t)$ and $B_{i}(t)(i=1,2)$.

It is well-known ([6], P. 484) that periodic continuously differentiable matrices $A_{k}(t)$ and $B_{k}(t)$ are representable as absolutely convergent Fourier series, namely,

$$
A_{k}(t)=\frac{\alpha_{0}^{k}}{2}+\sum_{j=1}^{\infty}\left[\alpha_{j}^{k} \cos (j r t)+\beta_{j}^{k} \sin (j r t)\right],
$$

RUSSIAN MATHEMATICS (IZ. VUZ) Vol. 57 No. 82013 


$$
B_{k}(t)=\frac{\widehat{\alpha}_{0}^{k}}{2}+\sum_{j=1}^{\infty}\left[\widehat{\alpha}_{j}^{k} \cos (j r t)+\widehat{\beta}_{j}^{k} \sin (j r t)\right], \quad k=1,2 ; \quad r=2 \pi / \sigma .
$$

In view of the absolute convergence of these series, there exists a positive integer number $\widehat{k}$ satisfying approximate equalities

$$
\begin{aligned}
& A_{k}(t) \approx \frac{\alpha_{0}^{k}}{2}+\sum_{j=1}^{\widehat{k}}\left[\alpha_{j}^{k} \cos (j r t)+\beta_{j}^{k} \sin (j r t)\right]=\widehat{A}_{k}(t), \\
& B_{k}(t) \approx \frac{\widehat{\alpha}_{0}^{k}}{2}+\sum_{j=1}^{\widehat{k}}\left[\widehat{\alpha}_{j}^{k} \cos (j r t)+\widehat{\beta}_{j}^{k} \sin (j r t)\right]=\widehat{B}_{k}(t), \quad k=1,2 .
\end{aligned}
$$

By replacing the time variable $t=\varepsilon_{n} \tau_{n}$ in system (1.7), (1.8), taking into account approximations (1.9), we obtain

$$
\begin{aligned}
& d x_{n+1}\left(\tau_{n}\right) / d \tau_{n}=\varepsilon_{n}\left[\widehat{A}_{1}\left(\varepsilon_{n} \tau_{n}\right) x_{n+1}\left(\tau_{n}\right)+\widehat{A}_{2}\left(\varepsilon_{n} \tau_{n}\right) x_{n}\left(\tau_{n}\right)\right. \\
& \left.+\widehat{B}_{1}\left(\varepsilon_{n} \tau_{n}\right) y_{n+1}\left(\tau_{n}\right)+\widehat{B}_{2}\left(\varepsilon_{n} \tau_{n}\right) y_{n}\left(\tau_{n}\right)\right], \\
& d y_{n+1}\left(\tau_{n}\right) / d \tau_{n}=e^{\varepsilon_{n} \tau_{n}}\left[A_{3} x_{n+1}\left(\tau_{n}\right)+A_{4} x_{n}\left(\tau_{n}\right)+B_{3} y_{n+1}\left(\tau_{n}\right)+B_{4} y_{n}\left(\tau_{n}\right)\right], \\
& 0 \leq \tau_{n} \leq \frac{\sigma}{\varepsilon_{n}}, \quad \varepsilon_{n}=\frac{e^{-n \sigma}}{\theta_{0}}, \lim _{n \rightarrow \infty} \varepsilon_{n}=0, \sum_{j=0}^{\infty} \varepsilon_{j}<\infty .
\end{aligned}
$$

Consider subsystem (1.10) with sufficiently large $n$. As is known ([7], P. 177), one can treat the totality of nonstationary terms in the right-hand side of this subsystem as linear perturbations "oscillating" with sufficiently small frequency, whose norms satisfy, respectively, bounds ([8], P. 259)

$$
\int_{s}^{\tau} \delta_{j}(s) d s \leq \widehat{\varepsilon}_{n}(\tau-s), \lim _{n \rightarrow \infty} \widehat{\varepsilon}_{n}=0, j=1,2
$$

where

$$
\delta_{1}\left(\tau_{n}\right)=\left\|x_{n+1}\left(\tau_{n}\right)\right\|, \quad \delta_{1}\left(\tau_{n}\right)=\left\|y_{n+1}\left(\tau_{n}\right)\right\|, \quad \delta_{2}\left(\tau_{n-1}\right)=\left\|x_{n}\left(\tau_{n}\right)\right\|, \quad \delta_{2}\left(\tau_{n-1}\right)=\left\|y_{n}\left(\tau_{n}\right)\right\| .
$$

Consequently, the first approximation subsystem for (1.10) with sufficiently large $n$ takes the form

$$
d x_{n+1}^{0}\left(\tau_{n}\right) / d \tau_{n}=\varepsilon_{n}\left\{\frac{\alpha_{0}^{1}}{2} x_{n+1}^{0}\left(\tau_{n}\right)+\frac{\alpha_{0}^{2}}{2} x_{n}^{0}\left(\tau_{n}\right)+\frac{\alpha_{0}^{3}}{2} y_{n+1}^{0}\left(\tau_{n}\right)+\frac{\alpha_{0}^{4}}{2} y_{n}^{0}\left(\tau_{n}\right)\right\},
$$

and the first approximation system corresponding to (1.10), (1.11) is the following differential-difference system

$$
\begin{aligned}
d x_{n+1}^{0}\left(\tau_{n}\right) / d \tau_{n} & =\varepsilon_{n}\left\{\frac{\alpha_{0}^{1}}{2} x_{n+1}^{0}\left(\tau_{n}\right)+\frac{\alpha_{0}^{2}}{2} x_{n}^{0}\left(\tau_{n}\right)+\frac{\alpha_{0}^{3}}{2} y_{n+1}^{0}\left(\tau_{n}\right)+\frac{\alpha_{0}^{4}}{2} y_{n}^{0}\left(\tau_{n}\right)\right\} \\
d y_{n+1}^{0}\left(\tau_{n}\right) / d t & =e^{\varepsilon_{n} \tau_{n}}\left\{A_{3} x_{n+1}^{0}\left(\tau_{n}\right)+A_{4} x_{n}^{0}\left(\tau_{n}\right)+B_{3} y_{n+1}^{0}\left(\tau_{n}\right)+B_{4} y_{n}^{0}\left(\tau_{n}\right)\right\} .
\end{aligned}
$$

Now let system (1.13) satisfy exponential stability conditions, i.e., inequalities (1.3), (1.4), and (1.5).

Theorem 1. If constant ("averaged”) matrices $\alpha_{0}^{k}, k=1, \ldots, 4$, satisfy conditions (1.3), (1.4), and (1.5), then system (1.7), (1.8) is exponentially stable.

Proof. Let us write the solution to system (1.10), (1.11) in the operator form

$$
\left(\begin{array}{c}
x_{n+1}^{0}\left(\tau_{n}\right) \\
y_{n+1}^{0}\left(\tau_{n}\right)
\end{array}\right)=T_{\tau_{n}, n}\left(\begin{array}{c}
x_{n}^{0}\left(\tau_{n}\right), y_{n+1}^{0}\left(\tau_{n}\right), y_{n}^{0}\left(\tau_{n}\right) \\
y_{n}^{0}\left(\tau_{n}\right), x_{n+1}^{0}\left(\tau_{n}\right), x_{n}^{0}\left(\tau_{n}\right)
\end{array}\right)
$$


where the linear shift operator obeys the formula

$$
T_{\tau_{n}, n}=\left\{\begin{array}{c}
T_{\tau_{n}, n}^{1} \\
T_{\tau_{n}, n}^{2}
\end{array}\right\}=\left\{\begin{array}{c}
U_{n}\left(\tau_{n}\right) u_{n}\left(\frac{\sigma}{\varepsilon_{n}}\right)+\int_{0}^{\tau_{n}} U_{n}\left(\tau_{n}-s\right) 0.5 \varepsilon_{n} \alpha_{0}^{2} u_{n}(s) d s \\
\quad+\int_{0}^{\tau_{n}} U_{n}\left(\tau_{n}-s\right) 0.5 \varepsilon_{n}\left(\alpha_{0}^{3} v_{n+1}(s)+\alpha_{0}^{4} v_{n}(s)\right) d s \\
V_{n}\left(\tau_{n}\right) v_{n}\left(\frac{\sigma}{\varepsilon_{n}}\right)+\int_{0}^{\tau_{n}} V_{n}\left(\tau_{n}, s\right) e^{\varepsilon_{n} s}\left(B_{4} v_{n}(s)\right. \\
\left.+A_{3} u_{n+1}(s)+A_{4} u_{n}(s)\right) d s
\end{array}\right.
$$

Here $U_{n}(\tau-s)$ is the fundamental solution matrix of the homogeneous system

$$
d \bar{u} / d \tau=0.5 \varepsilon_{n}\left[\alpha_{0}^{1} \bar{u}(\tau)+\alpha_{0}^{2} \bar{u}(\tau-\sigma)\right], \quad 0 \leq \tau \leq \sigma / \varepsilon_{n} ;
$$

owing to inequality (1.5)), it satisfies the bound

$$
\left\|U_{n}(\tau-s)\right\| \leq M_{1} e^{-\varepsilon_{n} \beta_{1}(\tau-s)}, M_{1}>0 .
$$

Analogously, the matrix

$$
V_{n}(\tau, s)=\exp \left\{B_{3} \frac{e^{\varepsilon_{n} \tau}-e^{\varepsilon_{n} s}}{\varepsilon_{n}}\right\}
$$

is the fundamental solution matrix of the homogeneous system

$$
d \bar{v} / d \tau=e^{\varepsilon_{n} \tau} B_{3} \bar{v}(\tau),
$$

and it also satisfies the bound

$$
\left\|V_{n}(\tau, s)\right\| \leq M_{2} \exp \left\{\frac{-\beta_{2}}{\varepsilon_{n}}\left(e^{\varepsilon_{n} \tau}-e^{\varepsilon_{n} s}\right)\right\}, M_{2} \geq 1 .
$$

Note that in view of bounds (1.15) and (1.16) the given operator $T_{\tau_{n}, n}$ is uniformly bounded [3, 4], i.e., $\left\|T_{\tau_{n}, n}\right\| \leq \bar{M}, \bar{M}>1$. Inequality (1.6) for the product of operators $T_{n_{j}, j}$ implies the bound [4]

$$
\begin{gathered}
\left.\left\|T_{\tau_{n}, n} \prod_{j=0}^{n-1} T_{s_{j}, j} w_{0}(s)\right\| \leq \widehat{M}_{0} q^{n} \sup _{\eta}\left\|x_{0}(\eta)\right\|+\underset{\eta}{\sup _{\eta}}\left\|y_{0}(\eta)\right\|\right), \\
w_{0}^{(\top)}(\eta)=\left\{x_{0}(\eta), y_{0}(\eta)\right\}, \quad \eta \in[-\sigma, 0], \quad q=e^{-\beta_{0} \sigma}, \quad 0<q<1, \widehat{M}_{0}>1 .
\end{gathered}
$$

Assume that $\varepsilon_{n}$ is sufficiently small for $n \geq N$ ( $N$ is a positive integer number).

Let us seek for a solution to the "perturbed" system (1.10), (1.11) stepwise. Denote "perturbations", respectively, by

$$
\begin{aligned}
f_{n+1}^{1}\left(\tau_{n}, x_{n+1}\left(\tau_{n}\right), x_{n}\left(\tau_{n}\right)\right) & =\left(\widehat{A}_{1}\left(\tau_{n}\right)-\alpha_{0}^{1} / 2\right) x_{n+1}\left(\tau_{n}\right)+\left(\widehat{A}_{2}\left(\tau_{n}\right)-\alpha_{0}^{2} / 2\right) x_{n}\left(\tau_{n}\right), \\
f_{n+1}^{2}\left(\tau_{n}, y_{n+1}\left(\tau_{n}\right), y_{n}\left(\tau_{n}\right)\right) & =\left(\widehat{B}_{1}\left(\tau_{n}\right)-\widehat{\alpha}_{0}^{1} / 2\right) y_{n+1}\left(\tau_{n}\right)+\left(\widehat{B}_{2}\left(\tau_{n}\right)-\widehat{\alpha}_{0}^{2} / 2\right) y_{n}\left(\tau_{n}\right) .
\end{aligned}
$$

We write the system solution in the integral (Cauchy) form, treating perturbing (oscillating) terms as inhomogeneities ([1], P. 162), and represent the solution in the operator form

$$
\begin{array}{r}
\left(\begin{array}{c}
x_{n+1}\left(\tau_{n}\right) \\
y_{n+1}\left(\tau_{n}\right)
\end{array}\right)=T_{\tau_{n}, n}\left(\begin{array}{c}
x_{n}^{0}\left(\tau_{n}\right), y_{n+1}^{0}\left(\tau_{n}\right), y_{n}^{0}\left(\tau_{n}\right) \\
y_{n}^{0}\left(\tau_{n}\right), x_{n+1}^{0}\left(\tau_{n}\right), x_{n}^{0}\left(\tau_{n}\right)
\end{array}\right) \\
\quad+I_{n}\left(f_{n+1}^{1}\left(\tau_{n}, x_{n+1}\left(\tau_{n}\right), x_{n}\left(\tau_{n}\right)\right), f_{n+1}^{2}\left(\tau_{n}, y_{n+1}\left(\tau_{n}\right), y_{n}\left(\tau_{n}\right)\right)\right) .
\end{array}
$$

Here $I_{n}$ is the linear integral operator defined as

$$
\begin{aligned}
I_{n}^{1}\left(f _ { n + 1 } ^ { 1 } \left(\tau_{n}, x_{n+1}\left(\tau_{n}\right),\right.\right. & \left.\left.x_{n}\left(\tau_{n}\right)\right), f_{n+1}^{2}\left(\tau_{n}, y_{n+1}\left(\tau_{n}\right), y_{n}\left(\tau_{n}\right)\right)\right) \\
= & \int_{0}^{\tau_{n}} U_{n}\left(\tau_{n}-s_{n}\right) \varepsilon_{n} f_{n+1}^{1}\left(s_{n}, x_{n+1}\left(s_{n}\right), x_{n}\left(s_{n}\right)\right) d s_{n}
\end{aligned}
$$

RUSSIAN MATHEMATICS (IZ. VUZ) Vol. 57 No. 82013 


$$
+\int_{0}^{\tau_{n}} U_{n}\left(\tau_{n}-s_{n}\right) \varepsilon_{n} f_{n+1}^{2}\left(s_{n}, y_{n+1}\left(s_{n}\right), y_{n}\left(s_{n}\right)\right) d s_{n} .
$$

Taking into account bounds (1.12), let us prove that terms containing values $f_{n+1}^{1}\left(\tau_{n}, x_{n+1}\left(\tau_{n}\right), x_{n}\left(\tau_{n}\right)\right)$ and $f_{n+1}^{2}\left(\tau_{n}, y_{n+1}\left(\tau_{n}\right), y_{n}\left(\tau_{n}\right)\right)$, have a higher order of smallness than unperturbed terms. Assume that $\tau_{n}>1,0 \leq s_{n} \leq k+\vartheta, k$ is a positive integer number, $\vartheta=$ const, and $0<\vartheta<1$. Then we get the following chain of inequalities:

$$
\begin{aligned}
& \left\|I_{n}^{1}\left(f_{n+1}^{1}\left(\tau_{n}, x_{n+1}\left(\tau_{n}\right), x_{n}\left(\tau_{n}\right)\right)\right)\right\| \\
& \leq \int_{0}^{\tau_{n}} M_{1} e^{-\varepsilon_{n} \beta_{1}\left(\tau_{n}-s_{n}\right)} \varepsilon_{n} \delta_{1}\left(s_{n}\right)\left\|x_{n+1}\left(s_{n}\right)\right\| d s_{n}+\int_{0}^{\tau_{n}} M_{1} e^{-\varepsilon_{n} \beta_{1}\left(\tau_{n}-s_{n}\right)} \varepsilon_{n} \delta_{2}\left(s_{n}\right)\left\|x_{n}\left(s_{n}\right)\right\| d s_{n} \\
& \leq M_{1} e^{-\varepsilon_{n} \beta_{i} \tau_{n}} \sup _{0 \leq s_{n} \leq \tau_{n}}\left\|x_{n+1}\left(s_{n}\right)\right\| \sum_{j=1}^{k+1} e^{\varepsilon_{n} \beta_{1} j} \int_{j}^{j+1} \varepsilon_{n} \delta_{1}(r) d r \\
& +M_{1} e^{-\varepsilon_{n} \beta_{1} \tau_{n}} \sup _{0 \leq s_{n} \leq \tau_{n}}\left\|x_{n}\left(s_{n}\right)\right\| \sum_{j=1}^{k+1} e^{\varepsilon_{n} \beta_{1} j} \int_{j}^{j+1} \varepsilon_{n} \delta_{2}(r) d r \\
& \quad \leq \varepsilon_{n} \widehat{\varepsilon}_{n} M_{1} \frac{e^{\varepsilon_{n} \beta_{1}}}{e^{\varepsilon_{n} \beta_{1}}-1} \sup _{0 \leq s_{n} \leq \tau_{n}}\left(\left\|x_{n+1}\left(s_{n}\right)\right\|+\left\|x_{n}\left(s_{n}\right)\right\|\right) .
\end{aligned}
$$

Note that $\lim _{n \rightarrow \infty} \frac{\varepsilon_{n}}{e^{\varepsilon_{n} \beta_{1}}-1}=\frac{1}{\beta_{1}}$.

$$
\text { If } \tau \leq 1 \text {, then }
$$

$$
\begin{array}{r}
M_{1} \int_{0}^{s} e^{-\varepsilon_{n} \beta_{1}\left(\tau_{n}-s_{n}\right)} \varepsilon_{n} \delta_{1}\left(s_{n}\right)\left\|x_{n+1}\left(s_{n}\right)\right\| d s_{n}+M_{1} \int_{0}^{s} e^{-\varepsilon_{n} \beta_{1}\left(\tau_{n}-s_{n}\right)} \varepsilon_{n} \delta_{2}\left(s_{n}\right)\left\|x_{n}\left(s_{n}\right)\right\| d s_{n} \\
\leq M_{1} \varepsilon_{n} \widehat{\varepsilon}_{n}\left(\sup _{0 \leq s_{n} \leq \tau_{n}}\left\|x_{n+1}\left(s_{n}\right)\right\|+\sup _{0 \leq s_{n} \leq \tau_{n}}\left\|x_{n+1}\left(s_{n}\right)\right\|\right) .
\end{array}
$$

In both cases the sum of these integrals does not exceed the value

$$
\mathbf{O}\left(\widehat{\varepsilon}_{n}\right)\left(\sup _{0 \leq s_{n} \leq \tau_{n}}\left\|x_{n+1}\left(s_{n}\right)\right\|+\sup _{0 \leq s_{n} \leq \tau_{n}}\left\|x_{n}\left(s_{n}\right)\right\|\right) .
$$

Now, estimating the value $\left\|I_{n}^{1}\left(f_{n+1}^{2}\left(\tau_{n}, y_{n+1}\left(\tau_{n}\right), y_{n}\left(\tau_{n}\right)\right)\right)\right\|$, we obtain an analogous bound, namely,

$$
\left\|I_{n}^{1}\left(f_{n+1}^{2}\left(\tau_{n}, y_{n+1}\left(\tau_{n}\right), y_{n}\left(\tau_{n}\right)\right)\right)\right\|=\mathbf{O}\left(\widehat{\varepsilon}_{n}\right)\left(\sup _{0 \leq s_{n} \leq \tau_{n}}\left\|y_{n+1}\left(s_{n}\right)\right\|+\sup _{0 \leq s_{n} \leq \tau_{n}}\left\|y_{n}\left(s_{n}\right)\right\|\right) .
$$

Since with $n<N$ system (1.10) (1.11) has bounded right-hand sides satisfying the Lipschitz conditions, we can estimate its solution with the help of the Bellman-Gronwall lemma ([8], P. 517). This solution $\left\{x_{n-1}, y_{n-1}\right\}^{\top}$ is, at least, bounded, i.e., there exists $L>0$ such that

$$
\max _{\tau_{n-2}}\left(\left\|x_{n-1}\left(\tau_{n-2}\right)\right\|+\left\|y_{n-1}\left(\tau_{n-2}\right)\right\|\right) \leq L \sup _{\eta}\left(\left\|\phi_{1}(\eta)\right\|+\left\|\phi_{2}(\eta)\right\|\right) .
$$

For the solution to the perturbed system (1.10), (1.11) defined by the initial vector function $\left\{x_{n-1}\left(\tau_{n-2}\right)\right.$, $\left.y_{n-1}\left(\tau_{n-2}\right)\right\}^{\top}$ with $n \geq N$, in view of bounds (1.17) and (1.18) (i.e., the smallness of perturbations), taking into accounts results obtained by us in [4], we get the bound

$$
\begin{gathered}
\max _{\tau_{n+j}}\left(\left\|x_{n+j+1}\left(\tau_{n+j}\right)\right\|+\left\|y_{n+j+1}\left(\tau_{n+j}\right)\right\|\right) \leq L_{1}\left(q_{1}\right)^{j} \max _{\tau_{n-2}}\left(\left\|x\left(\tau_{n-2}\right)\right\|+\left\|y\left(\tau_{n-2}\right)\right\|\right), \\
q<q_{1}<1, \quad L_{1}>1 .
\end{gathered}
$$

Formulas (1.19) and (1.20) imply an analogous exponential bound for any $x_{n}\left(\tau_{n-1}\right), y_{n}\left(\tau_{n-1}\right)$, $n=1,2, \ldots$. Furthermore, since

$$
\left\|A_{k}(t)-\widehat{A}_{k}(t)\right\|<\varepsilon,\left\|B_{k}(t)-\widehat{B}_{k}(t)\right\|<\varepsilon, \quad k=1,2
$$

( $\varepsilon$ is a sufficiently small positive number), the exponential stability of the first approximation system (1.13) implies the same property of system (1.10), (1.11) (in view of results obtained in [4]). 


\section{THE EXISTENCE OF AN ASYMPTOTICALLY PERIODIC SOLUTION}

Consider the inhomogeneous system

$$
\begin{gathered}
d \widehat{x}(t) / d t=A_{1}(t) \widehat{x}(t)+A_{2}(t) \widehat{x}(t-\sigma)+B_{1}(t) \widehat{y}(t)+B_{2}(t) \widehat{y}(t-\sigma)+f_{1}(t), \\
d \widehat{y}(t) / d t=\theta_{0} e^{t}\left[A_{3} \widehat{x}(t)+A_{4} \widehat{x}(t-\sigma)+B_{3} \widehat{y}(t)+B_{4} \widehat{y}(t-\sigma)+f_{2}(t)\right], \\
t \geq 0, \quad \theta_{0}>0, \sigma>0 .
\end{gathered}
$$

Here $f(t)(j=1,2)$ are periodic continuously differentiable functions with the period $T=\sigma$. Assume that the corresponding homogeneous system satisfies conditions of Theorem 1 . Then the solution to the perturbed homogeneous system (that corresponds to the first approximation system (1.13)) satisfies the bound

$$
\begin{gathered}
\left\|x\left(\tau_{n}\right)\right\|+\left\|y\left(\tau_{n}\right)\right\| \leq \widehat{L} \prod_{j=0}^{n}\left(q+\left\|\mathbf{O}\left(\varepsilon_{j}\right)\right\|+\|\mathbf{O}(\varepsilon)\|\right)\left(\sup _{\eta}\left\|\phi_{1}(\eta)\right\|+\sup _{\eta}\left\|\phi_{2}(\eta)\right\|\right), \\
\lim _{j \rightarrow \infty}\left(q+\left\|\mathbf{O}\left(\varepsilon_{j}\right)\right\|+\|\mathbf{O}(\varepsilon)\|\right)=q_{1}, \quad q<q_{1}<1, \sum_{j=1}^{\infty}\left\|\mathbf{O}\left(\varepsilon_{j}\right)\right\|<\infty .
\end{gathered}
$$

Taking into account the asymptotic behavior of the solution to the inhomogeneous perturbed system written in the integral form stepwise, in view of bound (2.2) we obtain

$$
\begin{aligned}
\left\|\widehat{x}\left(\tau_{n}\right)\right\|+\left\|\widehat{y}\left(\tau_{n}\right)\right\| \leq \widehat{L} \prod_{j=0}^{n}\left(q+\left\|\mathbf{O}\left(\varepsilon_{j}\right)\right\|+\|\mathbf{O}(\varepsilon)\|\right)\left(\sup _{\eta}\left\|\phi_{1}(\eta)\right\|+\sup _{\eta}\left\|\phi_{2}(\eta)\right\|\right) \\
+\widehat{L} \prod_{j=0}^{n-1}\left(q+\left\|\mathbf{O}\left(\varepsilon_{j}\right)\right\|+\|\mathbf{O}(\varepsilon)\|\right)\left(\max _{0 \leq t \leq \sigma}\left\|f_{1}(t)\right\|+\max _{0 \leq t \leq \sigma}\left\|f_{2}(t)\right\|\right) \\
+\widehat{L} \prod_{j=0}^{n-2}\left(q+\left\|\mathbf{O}\left(\varepsilon_{j}\right)\right\|+\|\mathbf{O}(\varepsilon)\|\right)\left(\max _{0 \leq t \leq \sigma}\left\|f_{1}(t)\right\|+\max _{0 \leq t \leq \sigma}\left\|f_{2}(t)\right\|\right)+\cdots \\
+\widehat{L}\left(\max _{0 \leq t \leq \sigma}\left\|f_{1}(t)\right\|+\max _{0 \leq t \leq \sigma}\left\|f_{2}(t)\right\|\right) \leq \widehat{L}_{1} \prod_{j=0}^{n}\left(q_{1}+\left\|\mathbf{O}\left(\varepsilon_{j}\right)\right\|\right)\left(\sup _{\eta}\left\|\phi_{1}(\eta)\right\|+\sup _{\eta}\left\|\phi_{2}(\eta)\right\|\right) \\
\quad+\frac{\widehat{L}_{1}}{1-q_{1}}\left(\max _{0 \leq t \leq \sigma}\left\|f_{1}(t)\right\|+\max _{0 \leq t \leq \sigma}\left\|f_{2}(t)\right\|\right), \quad \widehat{L}<\widehat{L}_{1}<\infty .
\end{aligned}
$$

This estimate means the boundedness of solutions $\left\{\left\|\widehat{x}\left(t, \phi_{1}(\theta)\right)\right\|,\left\|\widehat{y}\left(t, \phi_{2}(\theta)\right)\right\|\right\}$ to system $(2.1)$. Then (as follows from Theorem 1, namely, from a correlation analogous to (1.10)) $\lim _{t \rightarrow \infty} d \widehat{x}(t) / d t=0$. This allows us to study asymptotic properties of some partial solutions under additional conditions imposed on the right-hand sides of the inhomogeneous system (2.1).

The next theorem is a continuation of the study performed in [9].

Theorem 2. Let assumptions of Theorem 1 be fulfilled together with the following conditions:

1) there exists a matrix $\left(B_{3}+B_{4}\right)^{-1}$,

2) there exists a constant $m$-dimensional vector $\widehat{C}$ such that

$$
\left(A_{1}(t)+A_{2}(t)\right) \widehat{C}+f_{1}(t)=\left(B_{1}(t)+B_{2}(t)\right)\left[\left(B_{3}+B_{4}\right)^{-1}\left[\left(A_{3}+A_{4}\right) \widehat{C}+f_{2}(t)\right]\right] .
$$

Then system (2.1) has an asymptotically periodic (with a period $\sigma$ ) solution $\left\{\widehat{x}_{\sigma}, \widehat{y}_{\sigma}\right\}^{\top}$, where

$$
\widehat{x}_{\sigma}=\widehat{C}, \widehat{y}(t)=-\left(B_{3}+B_{4}\right)^{-1}\left[\left(A_{3}+A_{4}\right) \widehat{C}+f_{2}(t)\right] .
$$

Proof. System (2.1) corresponds to the inhomogeneous differential-difference system

$$
\begin{gathered}
d \widehat{x}_{n+1}(t) / d t=A_{1}(t) \widehat{x}_{n+1}(t)+A_{2}(t) \widehat{x}_{n}(t)+B_{1}(t) \widehat{y}_{n+1}(t)+B_{2}(t) \widehat{y}_{n}(t)+f_{1}(t), \\
\varepsilon_{n} d \widehat{y}_{n+1}(t) / d t=e^{t}\left[A_{3} \widehat{x}_{n+1}(t)+A_{4} \widehat{x}_{n}(t)+B_{3} \widehat{y}_{n+1}(t)+B_{4} \widehat{y}_{n}(t)+f_{2}(t)\right],
\end{gathered}
$$




$$
0 \leq t \leq \sigma, \widehat{y}_{n+1}(0)=\widehat{y}_{n}(\sigma), \widehat{x}(t) \approx \widehat{C}, n \geq N .
$$

Consider the second subsystem. Let $N$ be a sufficiently large positive integer number. Because of the presence of the small parameter $\varepsilon_{n}$ in the left-hand side of the subsystem, we seek for a partial solution in the form

$$
\widehat{y}_{\sigma}(t)=-\left(B_{3}+B_{4}\right)^{-1}\left[\left(A_{3}+A_{4}\right) \widehat{C}+f_{2}(t)\right] .
$$

Let $\widehat{x}_{n}(t)=\widehat{C}+x_{n}^{0}(t)$ and $\widehat{y}_{n}(t)=y(t)_{\sigma}+y_{n}^{0}(t)$. Then in view of (2.4) from the second subsystem in (2.3) we get

$$
\begin{gathered}
d x_{n+1}(t)^{0} / d t=A_{1}(t) x_{n+1}^{0}(t)+A_{2}(t) x_{n}^{0}(t)+B_{1}(t) y_{n+1}^{0}(t)+B_{2}(t) y_{n}^{0}(t), \\
\begin{array}{r}
\varepsilon_{n} d y_{\sigma}(t) / d t+\varepsilon_{n} d y_{n+1}^{0}(t) / d t \\
=e^{t}\left[A_{3} \widehat{C}+A_{4} \widehat{C}+A_{3} \widehat{x}_{n+1}^{0}(t)+A_{4} \widehat{x}_{n}^{0}(t)+B_{3} y_{\sigma}(t)+B_{4} y_{\sigma}(t)+B_{3} y_{n+1}^{0}(t)+B_{4} \widehat{y}_{n}^{0}(t)+f_{2}(t)\right] \\
=e^{t}\left[A_{3} \widehat{x}_{n+1}^{0}(t)+A_{4} \widehat{x}_{n}^{0}(t)+B_{3} y_{n+1}^{0}(t)+B_{4} \widehat{y}_{n}^{0}\right] .
\end{array}
\end{gathered}
$$

This system is inhomogeneous, and the vector function $\varepsilon_{n} d y_{\sigma}(t) / d t$ in it is vanishing (it tends to zero as $n \rightarrow \infty)$. Let us prove that the solution to this system $\left\{x_{n}^{0}(t), y_{n}^{0}(t)\right\}^{\top} \rightarrow 0$ as $n \rightarrow \infty$. Let $\widehat{q}=q+\mathbf{O}(\varepsilon)$, $q=e^{-\beta_{0} \sigma}$, and $q<\widehat{q}<1$. Without loss of generality, we assume that

$$
\widehat{q}>\mu: \mu=\delta \widehat{q}, 0<\delta<1 .
$$

Using the constant variation formula ([9], P. 157), we can write the solution to the perturbed inhomogeneous system on any step. We have

$$
\begin{aligned}
w_{n+k, \varepsilon}(t)=\widehat{T}_{n+k-1} \widehat{T}_{n+k-2} \ldots \widehat{T}_{n}\left(w_{n}(s)\right)+\varepsilon_{1} \widehat{T}_{n+k-1} \widehat{T}_{n+k-2} \ldots \widehat{T}_{n+1} d y_{\sigma}(t) / d t & \\
& +\varepsilon_{2} \widehat{T}_{n+k-1} \widehat{T}_{n+k-2} \ldots \widehat{T}_{n+2} d y_{\sigma}(t) / d t+\cdots+\varepsilon_{k} d y_{\sigma}(t) / d t
\end{aligned}
$$

Here $\widehat{T}_{n}$ is the shift operator similar to (1.19) acting in the Banach space $C_{2 m}[0, \tau]$ of continuous functions ([5], P. 124). In this space the solution to the unperturbed (linear) system is also representable in the operator form. Asymptotic properties of this operator [4] are analogous to properties of the operator $T_{n, \tau_{n}}$. Namely, the product of these operators (as follows from Theorem 1) satisfies the bound

$$
\begin{gathered}
\left\|\prod_{j=N}^{N+k} \widehat{T}_{j} w_{N-1}(s)\right\| \leq \widehat{L}_{2} \widehat{q}^{k}\left(\left\|x^{N-1}(t)\right\|_{\tau}+\left\|y_{N-1}(\tau)\right\|_{\tau}\right), \\
w_{N-1}^{(\top)}(t)=\left\{x^{N-1}(t), y^{N-1}(t)\right\}, \quad \widehat{L}_{2} \geq 1, \quad k=1,2, \ldots
\end{gathered}
$$

These operators are also uniformly bounded. Correlations (2.6) and (2.7) imply the inequality

$$
\begin{aligned}
& \max _{0 \leq t \leq \sigma}\left(\left\|x_{n+k}^{0}(t)\right\|+\left\|y_{n+k}^{0}(t)\right\|\right) \leq \widehat{L}_{2} \widehat{q}^{k} \max _{0 \leq t \leq \sigma}\left(\left\|x_{n}^{0}(t)\right\|+\left\|y_{n}^{0}(t)\right\|\right)+\widehat{L}_{2} \widehat{q}^{k-1} \max _{0 \leq t \leq \sigma}\left\|d y_{\sigma}(t) / d t\right\| \\
& \quad+\widehat{L}_{2} \varepsilon_{1} \widehat{q}^{k-2} \max _{0 \leq t \leq \sigma}\left\|d y_{\sigma}(t) / d t\right\|+\widehat{L}_{2} \varepsilon_{2} \widehat{q}^{k-3} \max _{0 \leq t \leq \sigma}\left\|d y_{\sigma}(t) / d t\right\|+\cdots+\widehat{L}_{2} \varepsilon_{k-1} \max _{0 \leq t \leq \sigma}\left\|d y_{\sigma}(t) / d t\right\| .
\end{aligned}
$$

Hence, taking into account (2.5), we obtain

$$
\begin{aligned}
& \max _{0 \leq t \leq \sigma}\left(\left\|x_{n+k}^{0}(t)\right\|+\left\|y_{n+k}^{0}(t)\right\|\right) \leq \widehat{L}_{2} \widehat{q}^{k} \max _{0 \leq t \leq \sigma}\left(\left\|x_{n}^{0}(t)\right\|+\left\|y_{n}^{0}(t)\right\|\right)+\widehat{L}_{2} \widehat{q}^{k-1} \max _{0 \leq t \leq \sigma}\left\|d y_{\sigma}(t) / d t\right\| \\
&+\widehat{L}_{2} \widehat{q}^{k-1} \frac{\delta}{\theta_{0}} \max _{0 \leq t \leq \sigma}\left\|d y_{\sigma}(t) / d t\right\|+\widehat{L}_{2} \widehat{q}^{k-1} \frac{\delta^{2}}{\theta_{0}} \max _{0 \leq t \leq \sigma}\left\|d y_{\sigma}(t) / d t\right\|+\cdots+\widehat{L}_{2} \widehat{q}^{k-1} \frac{\delta^{k-1}}{\theta_{0}} \max _{0 \leq t \leq \sigma}\left\|d y_{\sigma}(t) / d t\right\| \\
& \leq \widehat{L}_{2} \widehat{q}^{k} \max _{0 \leq t \leq \sigma}\left(\left\|x_{n}^{0}(t)\right\|+\left\|y_{n}^{0}(t)\right\|\right)+\frac{\widehat{L}_{2} \widehat{q}^{k-1}}{\theta_{0}(1-\delta)} \max _{0 \leq t \leq \sigma}\left\|d y_{\sigma}(t) / d t\right\| .
\end{aligned}
$$

Since values $\max _{0 \leq t \leq \sigma}\left(\left\|x_{n}^{0}(t)\right\|+y_{n}^{0}(t) \|\right), \max _{0 \leq t \leq \sigma}\left\|d y_{\sigma}(t) / d t\right\|$ are bounded, we conclude that

$$
\max _{0 \leq t \leq \sigma}\left(\left\|x_{n+k}^{0}(t)\right\|+\left\|y_{n+k}^{0}(t)\right\|\right) \rightarrow 0 \text { as } k \rightarrow \infty .
$$


Remark. Condition 2) of Theorem 2 may be fulfilled accurate to a vanishing vector function.

\section{AN EXAMPLE}

Consider the asymptotic behavior of a fourth-order system with the following parameters: the delay $\sigma=1$, matrices $A_{j}$ and $B_{j}, j=1, \ldots, 4$, with components

$$
\begin{gathered}
A_{1}=\left(\begin{array}{cc}
\sin p t & \cos p t \\
\sin p t & -\cos p t
\end{array}\right), A_{2}=\left(\begin{array}{cc}
-\cos p t & \sin p t \\
\sin p t & \sin p t
\end{array}\right), A_{4}=\left(\begin{array}{cc}
0.4 & 0.1 \\
0 & 0.4
\end{array}\right), \\
B_{1}=\left(\begin{array}{cc}
4 \cos p t & -\cos p t \\
-0.5 \cos p t & -\sin p t
\end{array}\right), B_{2}=\left(\begin{array}{cc}
0.3 \cos p t & -0.5 \cos p t \\
\sin p t & \sin p t
\end{array}\right), \\
B_{3}=\left(\begin{array}{cc}
-1 & 4 \\
0 & -1
\end{array}\right), B_{4}=\left(\begin{array}{ll}
1 & 2 \\
1 & 0
\end{array}\right)
\end{gathered}
$$

and the zero matrix $A_{3}$. Here $p=2 \pi / \sigma$. Furthermore, the vector function $f_{2}(t)=\left\{\cos ^{3} p t, \sin ^{3} p t\right\}^{\top}$,

$$
\begin{aligned}
& f_{1}(t)=\left(\begin{array}{cc}
4 \cos p t+0.3 \cos p t & -1.5 \cos p t \\
-0.5 \cos p t+\sin p t & 0
\end{array}\right) *\left(\begin{array}{cc}
-0.602 & -0.422 \\
-0.606 & -0.452
\end{array}\right) \\
& *\left[\left(\begin{array}{cc}
0.4 & 0.1 \\
0 & 0.4
\end{array}\right) *\left(\begin{array}{l}
1 \\
2
\end{array}\right)+\left(\begin{array}{c}
\cos ^{3} p t \\
\sin ^{3} p t
\end{array}\right)\right]-\left(\begin{array}{cc}
4 \cos p t+0.3 \cos p t & -1.5 \cos p t \\
-0.5 \cos p t+\sin p t & 0
\end{array}\right) *\left(\begin{array}{l}
1 \\
2
\end{array}\right) \\
& =\left(\begin{array}{c}
-0.217 \cos p t-1.68 \cos ^{4} p t-1.137 \cos p t \sin ^{3} p t \\
0.849 \cos p t-1.699 \sin p t+0.301 \cos ^{4} p t-0.62 \cos ^{3} p t \sin p t+0.211 \cos p t \sin ^{3} p t-0.422 \sin ^{4} p t
\end{array}\right) .
\end{aligned}
$$

Evidently, the vector function $f_{1}(t)$ satisfies condition 2) of Theorem 2. Asymptotic properties of the corresponding homogeneous system (more precisely, the corresponding first approximation system) are described rather thoroughly in [3]; namely, it is shown that the first approximation system is exponentially stable and (as follows from Theorem 1) so is the corresponding homogeneous system. Finally, the fourth-order system described by equalities (3.1) and (3.2) has an asymptotically periodic solution in the form

$$
x_{\sigma}=\left(\begin{array}{l}
1 \\
2
\end{array}\right), y_{\sigma}=\left(\begin{array}{l}
-0.9-0.067 \cos ^{3} p t-0.417 \sin ^{3} p t \\
-0.1-0.067 \cos ^{3} p t-0.017 \sin ^{3} p t
\end{array}\right) \text {. }
$$

\section{REFERENCES}

1. E. A. Barbashin, Introduction to the Theory of Stability (Nauka, Moscow, 1967; Wolters-Noordhoff, Groningen, The Netherlands, 1970).

2. B. G. Grebenshchikov, "The Asymptotic Behavior of the Linear Time-Delay System Solutions," Izv. Ross. Akad. Nauk. Teoriya i Sistemy Upravleniya, No. 2, 29-34 (2008).

3. B. G. Grebenshchikov and S. I. Novikov, "Instability of Systems with Linear Delay Reducible to Singularly Perturbed Ones,” Izv. Vyssh. Uchebn. Zaved. Mat., No. 2, 3-12 (2010)[Russian Mathematics (Iz. VUZ) 54 (2), 1-10(2010)].

4. B. G. Grebenshchikov, "The First-Approximation Stability of a Nonstationary System with Delay," Izv. Vyssh. Uchebn. Zaved. Mat., No. 2, 34-42 (2012) [Russian Mathematics (Iz. VUZ) 56 (2), 29-36 (2012)].

5. R. Bellman, and K. L. Cooke, Differential-Difference Equations (Academic Press, New York-London, 1963; Mir, Moscow, 1967). 
6. G. M. Fikhtengol'ts, Course of Differential and Integral Calculus (Mir, Moscow, 1966), Vol. 3 [in Russian].

7. Yu. A. Mitropol'skii, Method of Averaging in Nonlinear Mechanics (Kiev, 1971) [in Russian].

8. B. F. Bylov, R. E. Vinograd, D. M. Grobman, and V. V. Nemytskii, The Theory of the Indices of Lyapunov and Its Application to Stability Problems (Nauka, Moscow, 1967) [in Russian].

9. B. G. Grebenshchikov, "Existence of an Asymptotically Periodic Solution to a System with Delay," Izv. Ural'sk. Gos. Univ, No. 26. (Matematika i Mekhanika. Vyp. 5), 44-54 (2003).

10. A. Halanay and D. Wexler, Qualitative Theory of Pulse Systems (Editura Academiei, Bucharest, 1968; Mir, Moscow, 1971).

Translated by O. A. Kashina 